\title{
Beliefs Toward Smoking and COVID-19, and The Pandemic Impact on Smoking Behavior and Quit Intention: Findings from a Community-Based Cross-Sectional Study in Jordan
}

\author{
Ala'a B. Al-Tammemi ${ }^{1}$, Muna Barakat ${ }^{2}$, Doa'a B. Al-Tamimi ${ }^{3}$, Sami A. Alhallaq ${ }^{4}$, Dima \\ M. Al Hasan ${ }^{5}$, Ghena M. Khasawneh ${ }^{5}$, Khalil Abu Naqera ${ }^{6}$, Raghad M. Jaradat ${ }^{4}$, Fadi W. \\ Farah $^{7}$, Hindya O. Al-Maqableh ${ }^{8}$, Alaa Abuawad ${ }^{9}$, Bayan Othman $^{9}$, Zeinab Tarhini ${ }^{10}$, \\ Hamza Odeh ${ }^{11}$, Moawiah Khatatbeh ${ }^{8}$, Amal Akour ${ }^{12}$, Musheer A. Aljaberi ${ }^{13}$, and László \\ Róbert Kolozsvári ${ }^{1}$ \\ ${ }^{1}$ University of Debrecen \\ ${ }^{2}$ Applied Science Private University \\ ${ }^{3}$ Mutah University \\ ${ }^{4}$ Jordanian Royal Medical Services \\ ${ }^{5}$ Jordan University of Science and Technology \\ ${ }^{6}$ United Nations Relief and Works Agency for Palestine Refugees in the Near East Jordan \\ ${ }^{7}$ Jordan Ministry of Health \\ ${ }^{8}$ Yarmouk University Faculty of Medicine \\ ${ }^{9}$ Applied Science Private University Faculty of Pharmacy \\ ${ }^{10}$ University of Limoges Medical Faculty \\ ${ }^{11}$ Arabian Medical Relief (AMR) \\ ${ }^{12}$ The University of Jordan \\ ${ }^{13}$ Universiti Putra Malaysia
}

June 23, 2021

\begin{abstract}
Background: The relationship between smoking and Coronavirus Disease-2019 (COVID-19) is still topical with mixed epidemiological evidence. However, the pandemic may affect people's beliefs towards smoking as well as smoking behavior and quit intentions. Considering high smoking rates in Jordan, our current study aimed to assess the following points in a communitybased sample from Jordan: (i) the beliefs that surround smoking and COVID-19, (ii) the pandemic impact on smoking behavior and quit intention.

Methods: A cross-sectional study was conducted in Jordan utilizing an online questionnaire. The questionnaire comprised 13 items on sociodemographic, health, and smoking profiles, 14 items to assess beliefs surrounding COVID-19 and the use of combustible cigarettes (CC), waterpipe (WP), and electronic cigarettes (EC), and 12 items to assess the pandemic impact on smoking behavior and quit intention.

Results: Of 2424 survey respondents who participated in our study there were 1163 never-smokers, 1044 current smokers, and 217 ex-smokers. The mean age of participants was 35.2 years (SD:11.06). Most participants have shown anti-smoking beliefs with around $72.9 \%$ believed that WP smoking is related to the risk of contracting COVID-19. Also, $71.7 \%$ believed that smoking CC may worsen the COVID-19 clinical course, while $74.1 \%$ of respondents believed that smoking has no protective effect against COVID-19. During the pandemic, about $28.1 \%$ and $19.3 \%$ of current smokers reported increased or reduced smoking, respectively, and many smokers reported their willingness to quit smoking due to the pandemic. Besides, 459 current smokers have expressed their plans/intention to quit smoking during the pandemic, of whom $27.5 \%(\mathrm{n}=126)$ confirmed that the driving force for their decision is a COVID-19 related reason, such as self-protection $(\mathrm{n}=123)$ and protection of family
\end{abstract}


members $(=121)$ which were the most reported ones. Also, around 63 participants have successfully ceased smoking during the pandemic. However, only 22 of them reported that the main driving motivation of their successful quit attempt was the COVID-19 pandemic.

Conclusion: Most participants reported anti-smoking beliefs and attitudes during the pandemic. Nevertheless, the doubleedged effect of the pandemic on smoking habits should be carefully considered, and reliable anti-smoking measures should be strengthened and sustained in the country.

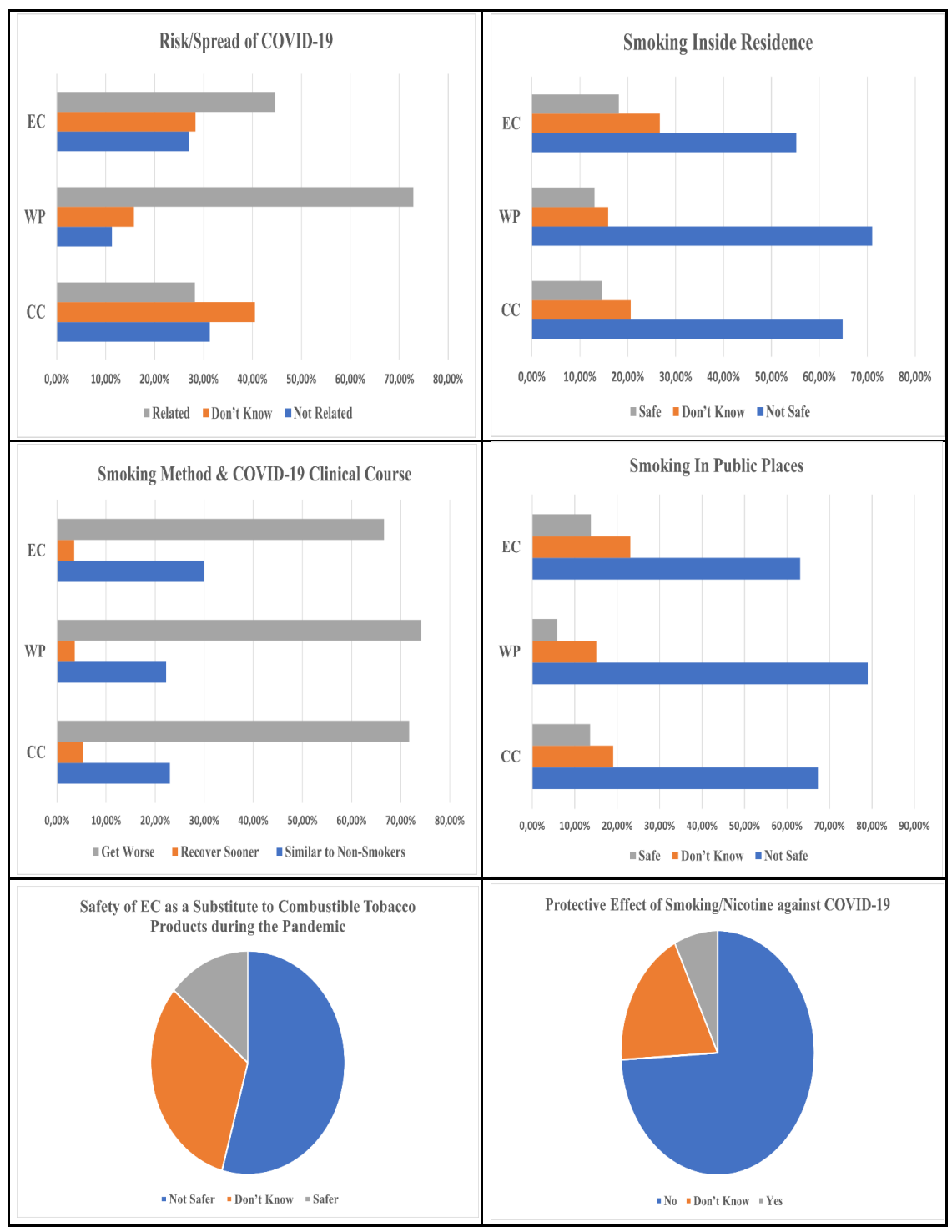



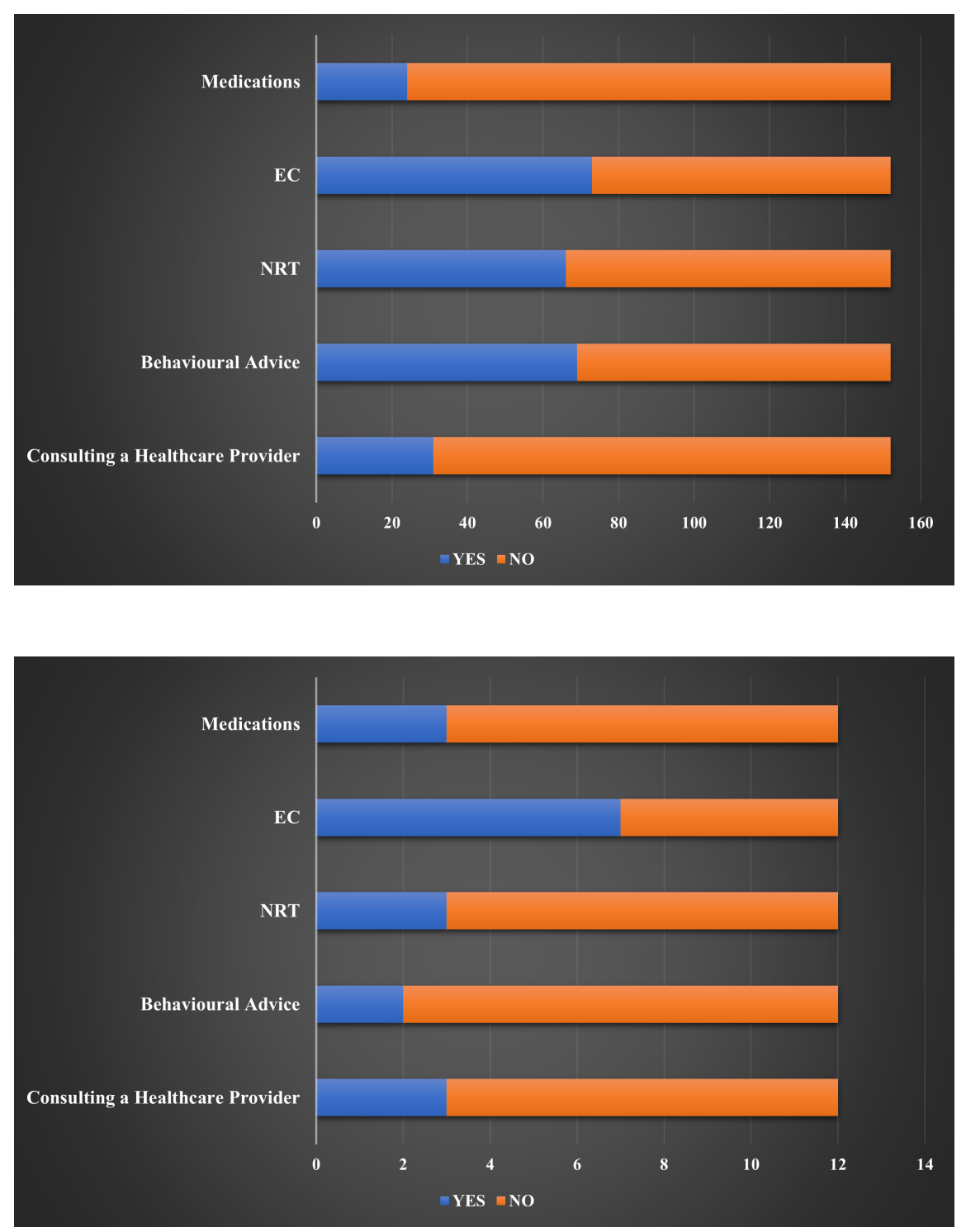

\section{Hosted file}

Table 1.docx available at https://authorea.com/users/412829/articles/524732-beliefs-towardsmoking-and-covid-19-and-the-pandemic-impact-on-smoking-behavior-and-quit-intentionfindings-from-a-community-based-cross-sectional-study-in-jordan

\section{Hosted file}

Table 2.docx available at https://authorea.com/users/412829/articles/524732-beliefs-towardsmoking-and-covid-19-and-the-pandemic-impact-on-smoking-behavior-and-quit-intentionfindings-from-a-community-based-cross-sectional-study-in-jordan

\section{Hosted file}

Table 3.docx available at https://authorea.com/users/412829/articles/524732-beliefs-towardsmoking-and-covid-19-and-the-pandemic-impact-on-smoking-behavior-and-quit-intentionfindings-from-a-community-based-cross-sectional-study-in-jordan 


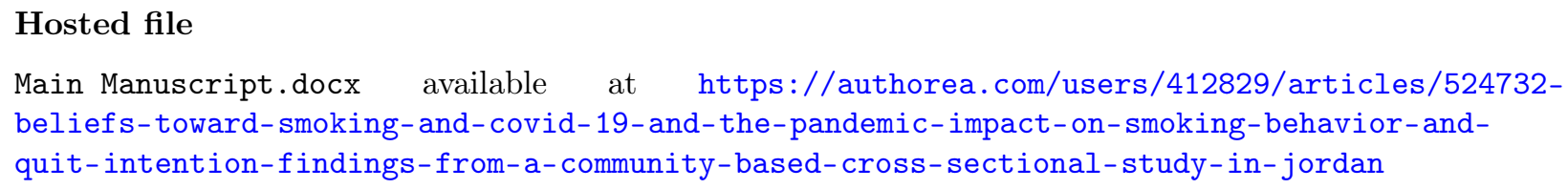

Beliefs Toward Smoking and COVID-19, and The Pandemic Impact on Smoking Behavior and Quit Intention: Findings from a Community-Based Cross-Sectional Study in Jordan

\begin{abstract}
Background: The relationship between smoking and Coronavirus Disease-2019 (COVID-19) is still topical with mixed epidemiological evidence. However, the pandemic may affect people's beliefs towards smoking as well as smoking behavior and quit intentions. Considering high smoking rates in Jordan, our current study aimed to assess the following points in a community-based sample from Jordan: (i) the beliefs that surround smoking and COVID-19, (ii) the pandemic impact on smoking behavior and quit intention.
\end{abstract}

Methods: A cross-sectional study was conducted in Jordan utilizing an online questionnaire. The questionnaire comprised 13 items on sociodemographic, health, and smoking profiles, 14 items to assess beliefs surrounding COVID-19 and the use of combustible cigarettes (CC), waterpipe (WP), and electronic cigarettes (EC), and 12 items to assess the pandemic impact on smoking behavior and quit intention.

Results: Of 2424 survey respondents who participated in our study there were 1163 never-smokers, 1044 current smokers, and 217 ex-smokers. The mean age of participants was 35.2 years (SD:11.06). Most participants have shown anti-smoking beliefs with around $72.9 \%$ believed that WP smoking is related to the risk of contracting COVID-19. Also, $71.7 \%$ believed that smoking CC may worsen the COVID-19 clinical course, while $74.1 \%$ of respondents believed that smoking has no protective effect against COVID19. During the pandemic, about $28.1 \%$ and $19.3 \%$ of current smokers reported increased or reduced smoking, respectively, and many smokers reported their willingness to quit smoking due to the pandemic. Besides, 459 current smokers have expressed their plans/intention to quit smoking during the pandemic, of whom $27.5 \%(\mathrm{n}=126)$ confirmed that the driving force for their decision is a COVID-19 related reason, such as self-protection $(\mathrm{n}=123)$ and protection of family members $(=121)$ which were the most reported ones. Also, around 63 participants have successfully ceased smoking during the pandemic. However, only 22 of them reported that the main driving motivation of their successful quit attempt was the COVID-19 pandemic.

Conclusion: Most participants reported anti-smoking beliefs and attitudes during the pandemic. Nevertheless, the double-edged effect of the pandemic on smoking habits should be carefully considered, and reliable anti-smoking measures should be strengthened and sustained in the country.

Keywords: COVID-19; Pandemic; Jordan; Smoking Behavior; Quit; Tobacco

\title{
1.INTRODUCTION
}

The Coronavirus Disease-2019 (COVID-19) pandemic has significantly impacted most life domains, including economy, politics, education, social life, travel, entertainment, and health services [1]. In an effort to retard the spread of the disease, most countries have applied several mitigation strategies including restrictions on international travel, closing universities and schools, and enforcing face-masking, physical distancing, and quarantine/lockdowns. Unfortunately, the influence of COVID19 induced stress and lockdowns came up with substantial socio-economic and psychological consequences [2-6]. Despite various mitigation 
measures, the COVID-19 related morbidity and mortality are still rising globally, with over 156 million confirmed cases and more than 3.2 million deaths as of May 08, 2021 [7]. In Jordan, a middle-income country in the Eastern Mediterranean Region (EMR), the situation is not different as the country has been severely afflicted by the pandemic with a total of 719233 confirmed cases and 9076 deaths as of May 08, 2021 [8]. The successive pandemic waves accompanied by many factors such as people's non-compliance to the recommended health precautions, pandemic fatigue, and the pandemic-induced psychologic and economic impacts were all believed to have negative impacts on the epidemic situation in Jordan [9-11]. Also, the successive pandemic waves have impacted the health system imposing a significant burden on healthcare institutions and the health workforce in the country $[9,12]$.

Many factors were linked to the severity and outcome of COVID-19 among patients, and one of those factors was smoking. Globally, tobacco use still represents a major threat to global public health with its proven effects in suppressing the immune system and increasing the risk of various respiratory and cardiovascular illnesses [13, 14]. Also, tobacco use may lead to a vicious cycle of poverty among families with limited financial resources $[15,16]$. A yearly estimation of more than 8 million deaths occurs due to tobacco-related diseases, of which 1 million deaths are due to second-hand smoking [15]. It is essential to stress the point that the majority of smokers and tobacco-induced morbidity and mortality are found in low- and middle-income countries [15].

The negative impacts of smoking on the respiratory and immune systems, and the linkage of smoking with the progression of various pulmonary diseases are well addressed in the literature [14, 17]. However, mixed epidemiological evidence has characterized the relationship between smoking and the progression/severity of COVID-19 [17-19]. Current epidemiological evidence suggests that smoking is linked to the severity and progression of COVID-19 [17, 20], while other studies reported lower-than-expected risks of COVID-19 among smokers [21-24]. A systematic review by Vardavas et al [18] reported a statistically significant association between smoking status and primary endpoints of admission to Intensive Care Unit (ICU), ventilator use, or death among COVID-19 patients. The review has shown that smokers were 1.4 times more likely to have severe symptoms of COVID-19, and approximately 2.4 times more likely to be admitted to an ICU, need mechanical ventilation, or die compared to non-smokers. Also, another systematic review and meta-analysis by Alqahtani et al [25] reported that $22 \%$ of current smokers and $46 \%$ of ex-smokers in the studies included had severe COVID-19 complications, and current smoking was associated with a higher mortality rate among COVID-19 patients.

Moreover, the physical, mental, and financial impacts accompanied the COVID-19 pandemic may exhibit an effect on current smokers, including their smoking habits as well as their intention to quit smoking. For example, during the pandemic in Turkey, smoking cessation rates have increased as reported in a study conducted by Tetik et al [26]. This finding might be as a result of the widespread fear that accompanied the pandemic and the infection as it is primarily a respiratory illness, given the possibility that people who smoke will be at higher risk of COVID-19 or more prone to a worse outcome.

Recent reports declared Jordan to be amongst the countries with the highest rates of smoking in the EMR and the world with an average daily consumption of 23 cigarettes per male smoker, surpassing the figures of Indonesia [27-29]. Besides, Jordan was ranked the sixth globally with a smoking prevalence of $70.2 \%$ among men and $10.7 \%$ among women [29]. Jordan has an extensive anti-tobacco history that started in the 1970s [30]. Despite the immense efforts, the country is found to have high smoking rates revealing an insufficient anti-tobacco policy implementation and monitoring. Moreover, the country has adopted the National Tobacco Control Strategy for 2017-2019 with the more stringent implementation of the WHO's MPOWER package (Monitor, Protect, Offer help, Warn, Enforce and Raise taxes), aiming to reduce tobacco usage by $30 \%$ by 2025 [31].

\section{STUDY RATIONALE AND AIMS}

The COVID-19 associated morbidity and mortality are expected to pose an impact on the attitudes of people 
towards smoking during the pandemic crisis. Besides, the pandemic-induced social, economic, and psychological impacts may affect smoking-related behaviors and attitudes among current smokers [32-35]. On the other hand, this pandemic might encourage tobacco users to quit smoking considering the reported association between smoking and adverse outcomes of COVID-19 compared to non-smokers [35-37]. Considering the importance of understanding people's beliefs about smoking and its implications on developing targeted interventions and smoking cessation programs, and in the line with the previously described pandemic impacts, the linkage between smoking and COVID-19, the high smoking rates in Jordan, and the paucity of studies that assessed this topic during the pandemic in the EMR, our current study aims were (i) to assess and explore the attitudes and beliefs that surround smoking/vaping and COVID-19 (ii) to assess the impacts of the pandemic on smoking behaviors and quit plans/attempts, in a community-based sample from Jordan.

\section{METHODS AND MATERIALS}

\subsection{Study Setting}

Our current study was conducted in Jordan, a middle-income country located in the Eastern Mediterranean Region. According to the latest statistics, the population count of Jordan is about 10.8 million [38]. The official language of the country is Arabic.

\subsection{Study Design and Participants}

This study was a descriptive quantitative cross-sectional survey that spanned from March 9, 2021, to March 16, 2021. Data were collected utilizing an anonymous self-administered online questionnaire that was developed using Google Form ${ }^{\circledR}$ (A secure cloud-based survey tool). The questionnaire was delivered in Arabic, the national language of Jordan. Considering the control measures of the COVID-19 pandemic (e.g., physical distancing) and to reach participants from various regions in the country in a time-efficient way, the decision was made by the research team to collect the data using a web-based (online) survey tool. We advertised our study on various social media forums including Facebook ${ }^{\circledR}$, WhatsApp ${ }^{\circledR}$, LinkedIn ${ }^{\circledR}$, and Twitter ${ }^{\circledR}$. Employing a snowball convenience sampling technique, participants were recruited and further encouraged to share the survey link with their family members and social network.

Eligible participants should have been at least 18 years or older, able to read and understand Arabic and reside in Jordan. The nature of our study, its objectives, and eligibility criteria for participation were explained in the introductory letter of our survey questionnaire. Participation was voluntarily and individuals who were interested to get involved in the study have given online informed consent by declaring their eligibility and accepting to fill out the questionnaire.There were no incentives/rewards upon participation.

\subsection{Assessment Tool and Measures.}

A structured questionnaire was developed by the authors after reviewing relevant and related literature [19, 35, 46-48, 37, 39-45]. The questionnaire comprised 39 single-answer items that were distributed over three sections as the following:

\subsubsection{Sociodemographic, Health, and Smoking Profiles}

The first section included 13 items that solicited data on the sociodemographic profile, health profile and smoking profile of participants. The sociodemographic data included age (in years), gender (female, male or prefer not to say), marital status (single or married), educational level (up to secondary school or higher education), residence area (rural or urban), employment status (unemployed, employed in the health sector, 
employed in non-health sector), and the number of household members (less than 3 persons, 3-5 persons or 6 persons and more).

In the health profile, we asked the participants to report their physical activity based on the WHO recommendation of whether they maintain at least 150 minutes of moderate-intensity aerobic activity in a week (Yes or No), history of chronic diseases like hypertension, diabetes, chronic respiratory diseases or any other chronic illnesses (Yes or No), history of laboratory-confirmed COVID-19 infection (Yes or No), and history of COVID-19 infection among family members in the same house (Yes or No).

Regarding smoking profile, participants were requested to report their current smoking status (past 30day use) regarding combustible cigarettes (CC), waterpipe (WP), and electronic cigarettes (EC) which are the most popular smoking methods in Jordan. Accordingly, our study sample is represented by 1163 never-smokers, 1044 current smokers, and 217 ex-smokers.

\subsubsection{Beliefs about Smoking and COVID-19}

The second section of the questionnaire included 14 items that aimed to assess the participants' attitudes and beliefs surrounding various smoking methods and COVID-19. The participants were instructed to answer these items based on their beliefs and opinions. The 14 items of this section were adapted from a study after obtaining the authors' permission [19]. The 14 items were distributed as the following: three items on the relationship between smoking CC, WP, or EC and risk of contracting COVID-19 (related, not related or I do not know), three items on the safety of smoking CC, WP or EC inside the residence place during the pandemic crisis (safe, not safe, I do not know), three items on the safety of smoking CC, WP or EC in public places during the pandemic crisis (safe, not safe, I do not know), one item on the safety of EC as a substitute to any other form of smoking products during the pandemic (safe, not safe, I do not know), three items on the general outcome of a CC, WP or EC smoker in case of contracting COVID-19 infection (similar to non-smokers, recover sooner, get worse). Lastly, the respondents were asked whether they believe that smoking or nicotine use could provide protection against COVID-19 (Yes, No, or I do not know).

\subsubsection{Smoking Behavior and Quit Plans.}

The third section of our survey questionnaire assessed the impacts of the COVID-19 pandemic on smoking behavior and quit attempts/plans through 12 items that targeted both, current smokers, and ex-smokers with six items for each. Current smokers were asked about how did the pandemic impact their smoking habits in comparison to their habits before the pandemic crisis (no change, increased consumption, decreased consumption, shifting to another smoking method, in an active quitting trial, or the initiation of smoking), and participants were requested to choose the most applicable scenario. Also, participants were asked if they consider/plan to quit smoking during the pandemic (Yes, No), if they intend to use smoking cessation tools (Yes, No), types of smoking cessation tools they intend to use (all that applies from: consulting a healthcare provider, behavioral advice, Nicotine Replacement Therapy (NRT), medications or using EC ), the primary motivation for planning to quit smoking (COVID-19 related reasons, or reasons not related to COVID-19), and the main driving reasons to quit smoking due to COVID-19 (all that applies from: to protect myself, to protect my family, due to pandemic measures such as mask-wearing and closure of cafes or due to economic impacts/financial constraints induced by the pandemic). In the same section, ex-smokers were asked to report if they have successfully quitted smoking during COVID-19 or before the pandemic (yes, no), if their successful quit attempts were due to COVID-19 related reasons or not, the main driving reasons behind their decision to quit smoking due to COVID-19 issues, their prospective plan concerning their smoking cessation (sustaining it even after the pandemic is over, or resuming smoking after the pandemic is over), if they have used smoking cessation tools (yes, no), and types of smoking cessation tools used (response choices were similar to those provided for current smokers). 


\subsection{Face/Content Validity and Pilot-Testing}

The questionnaire was initially developed in English and evaluated by two academics in the field of tobacco research for content and face validity. Then, it was translated into Arabic by two independent academic translators using translation and back-translation techniques. To ensure clarity and understandability of the questionnaire's items among the general population, it was piloted on 50 participants from various academic and professional backgrounds, and linguistic refinements were made as needed based on the feedback from the pilot-testing phase. The pilot responses were not included in the final analyses.

\subsection{Sample Size}

The required sample size was estimated to be 385 participants based on the Raosoft ${ }^{\circledR}$ software using a confidence level of $95 \%$, response distribution of $50 \%$, a margin of error of $5 \%$. However, aiming at gathering a more comprehensive view regarding individuals' beliefs and attitudes, and considering that randomized sampling was not feasible, we were committed to including as many participants as we can. Thus, a total of 2424 participants have enrolled in our study, considering the use of a time-efficient and widely accessible tool by most people in Jordan, i.e., an internet survey.

\subsection{Data Management and Analyses}

Completed responses were extracted from Google Form ${ }^{\circledR}$ as an Excel sheet for quality check, data cleaning, and coding. Then, the Excel sheet was exported into Statistical Package for Social Sciences version 24.0 (SPSS Inc., Chicago, IL, USA) for further statistical analyses. Mean and standard deviation was used to describe numerical variables, while categorical variables were reported as frequency and percentages. Pearson's Chi-square test was employed to assess significant differences between smoking groups (never-smokers, ex-smokers, and current smokers) according to their sociodemographic profile, health profile, and beliefs surrounding smoking. Moreover, we estimated the adjusted Odds Ratio (aOR) and 95\% Confidence Interval (95\% CI) for smoking-related attitudes/beliefs using multinomial logistic regression, with smoking status (never-smokers, ex-smokers, and current smokers/reference) as a dependent outcome variable, while adjusting for sociodemographic and health factors. A $p<0.05$ was implemented for statistical significance.

\subsection{Ethical Considerations}

The protocol of this study was reviewed and approved by the institutional review board at [anonymized for peer review] (Ref. Anonymized for peer review). In addition, the study was conducted conforming to the ethical standards of the Declaration of Helsinki. The survey questionnaire ensured the confidentiality and anonymity of the study participants. Participation was voluntarily and online written informed consent was obtained from all participants as a pre-requisite before answering the survey items.

\section{RESULTS}

\subsection{Sociodemographic, Health, and Smoking Profile of Participants}

A total of $\mathbf{2 4 2 4}$ participants have consented and responded to our survey questionnaire. The mean age of the overall cohort was 35.2 years (SD:11.06). Female participants slightly predominated the sample $(\mathrm{n}=1256,51.8 \%)$. Most participants were married $(\mathrm{n}=1471,60.7 \%)$, with college/university education $(\mathrm{n}=2164,89.3 \%)$, employed in non-health sector $(\mathrm{n}=949,39.2 \%)$, living in urban areas $(\mathrm{n}=2122,87.5 \%)$, and residing with three to five persons in the same living place $(\mathrm{n}=1398,57.7 \%)$. Of the overall sample, $29.8 \%$ $(\mathrm{n}=723)$ of the participants were found to practice sufficient physical activity, while around $17.4 \%(\mathrm{n}=421)$ of participants reported having a chronic disease. Those who got infected with COVID-19 comprised 27.5\% 
$(\mathrm{n}=666)$ of participants, and $35.9 \%(\mathrm{n}=870)$ have reported at least one household member (living in the same house) who suffered from COVID-19. Regarding smoking status, around 48.0\% (1163/2424), 8.9\% $(217 / 2424)$, and $43.1 \%(1044 / 2424)$ of participants were identified to be never-smokers, ex-smokers, and current smokers, respectively. Table 1 shows a detailed presentation of the participants' sociodemographic and health profile classified per smoking status.

\subsection{Attitudes and Beliefs Toward Smoking and COVID-19}

Concerning smoking and the risk of contracting COVID-19, $72.9 \%$ of the study participants believed that WP smoking is related to the risk of COVID-19, while around $44.6 \%$ and $38.2 \%$ of respondents believed that $\mathrm{CC}$ and EC use are related to the risk of COVID-19 infection, respectively. Also, a significant proportion of participants believed that smoking CC, WP, or EC inside the residence place is not safe $(64.9 \%, 71.0 \%$, and $55.2 \%$, respectively), compared to using the same smoking products in public places $(67.3 \%, 79.0 \%$, and $63.1 \%$, respectively). Never-smokers were more likely to believe that smoking CC, WP, and EC are not safe inside residence places and in public places as well.

Moreover, around $71.7 \%$ believed that CC smokers would have a worse COVID-19 clinical course compared to those who do not smoke, and this finding was closely similar to the beliefs surrounding WP and EC usage. On the other hand, about one-quarter of respondents believed that smokers of CC, WP, or EC would have the same COVID-19 clinical course as that for non-smokers (23.0\%, 22.2\%, and 29.9\%). Similarly, never-smokers were more likely to believe that smoking CC, WP, or EC may worsen the COVID-19 illness. See Figure 1 and Table 2.

When we asked the participants about their beliefs toward EC as a substitute to other forms of smoking during the pandemic crisis, more than half $(54.3 \%)$ responded that EC is not a safer option according to their opinion. Besides, $74.1 \%$ of respondents believed that the use of tobacco products/nicotine does not provide a protective effect against COVID-19, and instead, they can worsen the disease course. See Figure 1 and Table 2.

\subsection{Findings of the Multinomial Regression Analysis}

As described in the data analysis section, a multinomial regression analysis was employed to assess the adjusted odds ratio (aOR) and $95 \%$ confidence interval (96\% CI) of attitudes/beliefs that surround smoking during the COVID-19 pandemic. Smoking status was treated as an outcome variable with current smokers as a reference category while adjusting for sociodemographic and health factors.

Regarding smoking and risk of COVID-19, the multinomial regression analysis revealed that never-smokers and ex-smokers were less likely to believe that smoking CC is not related to the risk of COVID-19 infection and spread ( $\mathrm{aOR}=0.48,95 \%$ CI: $0.33-0.69$; and $\mathrm{aOR}=0.57,95 \% \mathrm{CI}: 0.337-0.964$, respectively). On the other hand, never-smokers had higher odds in believing that WP smoking is not related to the risk of COVID-19 infection (aOR=1.872, 95\% CI:1.078 - 3.251). Ex-smokers were more likely to believe that EC use is not related to the risk of COVID-19 infection and spread (aOR=1.792, 95\% CI:1.021 - 3.146).

In the domain of smoking inside residence places and in public places, ex-smokers had lower odds and were less likely to believe that smoking $\mathrm{CC}$ inside residence place is safe ( $\mathrm{aOR}=0.33,95 \%$ CI:0.155 - 0.720), and never-smokers were more likely to believe that smoking WP in public places is not safe $(\mathrm{aOR}=1.83,95 \%$ CI: $1.156-2.896)$.

Besides, never-smokers had higher odds in believing that smoking CC would worsen the COVID-19 clinical course ( $\mathrm{aOR}=3.501,95 \% \mathrm{CI}: 1.970$ - 6.222). Both never-smokers and ex-smokers were more likely to believe that smoking/nicotine use does not provide a protective effect against COVID-19 (aOR=1.88, 95\% CI:1.389 - 2.545; and aOR=1.85, 95\% CI:1.162 - 2.927, respectively). Regarding the use of EC as a substitute to other forms of smoking during the pandemic, never-smokers were less likely to believe that EC could be a safer 
substitute (aOR=0.53, 95\% CI:0.333 - 0.830), and ex-smokers were more likely to believe that EC use is not a safer substitute as well $(\mathrm{aOR}=1.70,95 \%$ CI:1.059 - 2.736). See Table 3

\subsection{Impacts of the Pandemic on Smoking Behavior and Quit Plans among Current Smokers}

Among current smokers $(\mathrm{n}=1044), 42.4 \%(\mathrm{n}=443)$ have reported no change in their smoking behavior in general, while around $28.1 \%(\mathrm{n}=293)$ and $19.3 \%(\mathrm{n}=202)$ have reported increased consumption, or reduced consumption, respectively. Also, $2.6 \%(\mathrm{n}=27)$ and $1.2 \%(\mathrm{n}=13)$ reported shifting to another smoking method, and initiation of smoking, respectively. Regarding quit plans, around $6.3 \%(\mathrm{n}=66)$ expressed being in current active trials to quit smoking. When current smokers were asked if they have near-future plans/intention to quit smoking during the pandemic crisis (apart from those who were in an active quitting trial), around $44.0 \%(\mathrm{n}=459)$ of current smokers answered yes, of whom 152 participants cited their plans to use various smoking cessation tools with behavioral advice (45.4\%) and using EC (48.0\%) were the most reported items (See Figure 2). Besides, of the 459 current smokers who expressed their plans to quit smoking during the pandemic, around $27.5 \%(\mathrm{n}=126)$ confirmed that the primary driving force for their intention to quitting smoking is a COVID-19 related issue, of which self-protection $(\mathrm{n}=123)$ and protection of family members $(=121)$ were the most reported reasons, followed by pandemic measures such as mask-wearing and lockdowns $(\mathrm{n}=63)$ and pandemic-induced financial constraints $(\mathrm{n}=67)$.

\subsection{Impacts of the Pandemic on Successful Quitters}

Of the total number of ex-smokers who participated in our study $(\mathrm{n}=217), 63$ participants have reported their successful quit attempts during the pandemic crisis, while the rest were successful quitters before the beginning of the pandemic. Of the 63 successful quitters during the pandemic, only 12 have used smoking cessation tools, mostly EC. See Figure 3.

Besides, of the 63 successful quitters during the pandemic, only 22 have reported that the main driving motivation of their successful quit attempts was COVID-19 related issue, with self-protection and protection of family members were the most frequent reasons ( $n=22$ and $n=20$, respectively), followed by pandemic measures such as mask-wearing and lockdowns $(\mathrm{n}=9)$ and pandemic-induced financial constraints $(\mathrm{n}=6)$. Moreover, of the COVID-19 motivated ex-smokers $(\mathrm{n}=22), 19$ ex-smokers reported that they will sustain cessation even after the pandemic is over, while 3 have expressed their intention to resume smoking after the pandemic is over.

\section{DISCUSSION}

Life changes induced by the COVID-19 pandemic were remarkable, characterizing this pandemic as one of the most devastating events globally. Alongside the pandemic-induced repercussions that surrounded the global politics, economy, health sector, education, labor markets, travel, and social life, many changes in the individuals' lifestyle and habits were also a remark of this global crisis. The COVID-19 precautionary measures including lockdowns, physical distancing, mask-wearing, frequent hygienic practices, avoiding crowdedness, and many other restrictions on social life have impacted individuals' day-to-day activities.

The negative impacts of smoking on the respiratory and immune systems and the linkage of smoking with the progression of various pulmonary diseases are well addressed in the literature [17]. However, a controversy has characterized the relationship between smoking and the COVID-19, especially at the early stage of the COVID-19 outbreak. Many speculations surrounded the effects of smoking on COVID19, considering the biological effect of smoking and nicotine on the expression of Angiotensin-Converting Enzyme 2 (ACE2) on the pulmonary tissue and other tissues [21], which act as a potential entry receptor for SARS-CoV-2. Recent evidence prior to the COVID-19 reported a down-regulatory effect of nicotine on 
the expression of ACE2 receptor [49, 50]. On the other hand, evidence from studies during the COVID-19 pandemic revealed an up-regulatory effect of smoking and nicotine on ACE2 receptor [51-53]. Up-regulation of ACE2 was linked with higher susceptibility to COVID-19 infection [54]. As current smokers have higher gene expression of ACE2 compared to never-smokers and ex-smokers, a possible association between smoking and risk of COVID-19 infection was advocated [54]. On the other side, it was hypothesized that smoking and nicotine possess a protective effect against COVID-19 based on some evidence from epidemiological studies that involved hospitalized COVID-19 patients [21]. Also, other studies have reported a decreased risk of testing positive for SARS-CoV-2 among current smokers [22, 23]. However, these findings were criticized to have numerous biases [55].

Our study's first aim was to assess the beliefs surrounding various methods of smoking amid the COVID-19 pandemic in a study sample from the Jordanian community. Different beliefs were expressed by the participants regarding the relationship between smoking and many domains of COVID-19 including risk/spread of infection, the safety of smoking inside residences and in public places, COVID-19 clinical outcome, EC as a substitute to other tobacco products, and protective effect of smoking/nicotine. Of the overall cohort, around $38.2 \%, 72.9 \%$, and $44.6 \%$ of the participants believed that CC, WP, and EC are related to the risk of contracting COVID-19, with WP smoking being on the top of the list.

WP smoking is highly prevalent worldwide with an estimated 100 million daily users globally [56]. The popularity of WP smoking is possibly grounded in the attractiveness of flavored WP tobacco (Moassel or Maassel), the usability of WP device in smoking illicit drugs like Cannabis/Marijuana, and the social implications as this smoking method is prominent in social gatherings. Also, WP smoking is widely spread among young adults and youths in Arab states, where most cafés and restaurants provide WP service, and many individuals own a WP device at home. In general, the possibility of transmitting various infectious microbial agents through waterpipe usage is high, especially in cafés where cleaning and washing of WP devices after each customer's use is not feasible, and where sharing the same WP device with multiple users is also common practice [56]. Bacterial, viral and fungal microbes were reported in the literature to be transmitted to users through WP devices, such as Mycobacterium Tuberculosis, Helicobacter Pylori, Hepatitis C virus, Epstein-Barr virus, Herpes Simplex virus, Respiratory viruses, and even respiratory fungal pathogens (e.g. Aspergillus) [56-62]. These facts about WP and the associated infectious risks have forced many countries to enforce a ban on the use of WP in public places during the pandemic [63].

Additionally, a notable proportion of participants believed that various smoking methods (CC, WP, EC) are not safe to be used inside the residence place, with closely similar findings regarding their usage in public places as well. However, WP received the highest safety concerns among the respondents. Besides, most participants $(71.7 \%)$ expressed that that being a CC smoker may worsen the COVID-19 clinical course compared to those who do not smoke, with closely similar concerns surrounding WP $(74.1 \%)$ and EC $(66.7 \%)$ usage. Several recent studies addressed an association between smoking and the severity of COVID-19. Reddy et al (2021) reported in their systematic review and meta-analysis that current smokers had an increased risk of severe and critical COVID-19 with risk ratios of 1.80, and 1.98, respectively [64]. They also found that COVID-19 patients with a smoking history had an increased risk for severe or critical COVID-19, in-hospital mortality, disease progression, and need for mechanical ventilation [64].

On the contrary, a large-scale ecological study across 38 European countries that aimed to examine different factors associated with the number of COVID-19 cases per million people at a specific time-point revealed a significant negative relationship between the prevalence of smoking among the adult population and COVID19 cases [40]. In line with this finding, a systematic review by Farsalinos et al (2020) that examined the prevalence of current smoking among hospitalized COVID-19 patients in China, found a surprisingly low smoking prevalence among those patients, and this could be hypothetically linked to the immunomodulatory effect of nicotine [21]. However, this finding was criticized to have numerous biases [55]. In our study, $74.1 \%$ of respondents believed that the use of tobacco products/nicotine does not provide a protective effect against COVID-19, and instead, they believed that tobacco/nicotine may have a negative consequence on the clinical outcome of COVID-19. Never-smokers and ex-smokers were more likely to have this belief compared 
to current smokers in our study.

Moreover, EC as a novel smoking method is gaining popularity worldwide due to its appealing flavors and the commercial claims of their benefits as a smoking cessation tool, despite the controversial scientific evidence [65-68]. In Jordan, EC use is increasing as the government has recently legalized the importation, distribution, and sale of EC in various retail stores in the country [65, 67, 69]. In a national survey that was conducted in 2019 in Jordan, and among the 1820 participants involved, the prevalence of exclusive EC use and dual-use was estimated to be $11.7 \%$, and $4.0 \%$, respectively [70]. Another recent online crosssectional survey in Jordan that included 1536 participants found that around 18\% of the study sample were EC users, and its usage was more prominent among males and young age groups [71]. In our study, 54.3\% of participants reported their belief that EC is not a safe option to replace combustible tobacco products during the pandemic crisis, while around $13.8 \%$ believed EC is a safer option compared to WP or CC. Most believers in EC safety were current smokers. These EC-related beliefs may have been impacted by the pandemic crisis, and this was explored by a mixed-method study that was carried out in the United States [42]. Among these impacts were the perceived COVID-19 health risks that may be related to the inhaled toxic substances found in EC vapor and their effects on the lungs.

Concerning our study's second aim, the majority of current smokers $(57.6 \%, 601 / 1044)$ have reported a change in their smoking habits during the pandemic crisis. Most participants reported an increased consumption or reduced consumption. Increased tobacco use during the pandemic has been also reported in recent studies $[39,41,46]$. Besides, many current smokers in our study have reported their intention to quit smoking during the pandemic, which was also one of the findings of various studies during the pandemic crisis $[37,41]$. Various COVID-19 related reasons were cited by quit planners during the pandemic (among current smokers) and successful quitters during the pandemic (among ex-smokers). These reasons may reflect the profound effects of the pandemic crisis on individual's lifestyles and habits. Additionally, most successful quitters due to COVID-19 in our study have reported their willingness to sustain smoking cessation after the pandemic is over, which is also in line with the findings of a study that was conducted in Iran [19].

\section{STUDY IMPLICATIONS AND LIMITATIONS}

Our present study sheds the light on an important yet under-reported topic that is related to COVID-19 and smoking in Jordan. Various beliefs have surrounded smoking in its most common forms in the country, namely, combustible cigarettes, waterpipes, and electronic cigarettes. Despite the controversial evidence about the effect of smoking on COVID-19 during the early stages of the pandemic crisis, most recent studies pointed to a significant connection between smoking and the risk of COVID-19 progression, hospitalization, and mortality. In Jordan, a country that is ranked the sixth globally in the smoking rate, where anti-smoking policies are present but weakly enforced, where smoking has socio-cultural influences, and where tobacco industry front groups have significant interference in tobacco policymaking in the country, it is imperative to take the benefit of the pandemic crisis to advocate more stringent implementation of anti-tobacco measures in the community. Also, it is crucial to raise community awareness about the dangers of smoking in general and in relation to the COVID-19 utilizing mass media messages and to encourage smoking cessation using scientifically valid quitting tools. Although the pandemic may encourage current smokers to reduce their consumption or to quit, there is a possibility for the opposite scenario where pandemic-induced stress may lead to increased smoking [39], or even initiation of smoking which was noticed among few participants in our study. Therefore, public health decision-makers should be aware of the double-edged effect of the COVID-19 pandemic on tobacco use, and appropriate measures should be taken to prevent further escalation of smoking rates in Jordan, considering the severe economic and psychological impacts that afflicted most people in the country.

Our study has limitations that should be considered when interpreting the results. These include (i) using non-probability sampling that may limit the generalizability of our findings, (ii) using an online survey which also impacts the representatives of the study sample as this tool targets people with sufficient level of digital 
literacy and access to the internet, (iii) the cross-sectional nature of our study in which exploring cause-effect relationship is not feasible, (iv) the study was limited to Arabic-speaking individuals in Jordan, and lastly, (v) our questionnaire items represented self-reporting states; thus, recall bias might have an effect on the results. Nevertheless, we encourage nationally representative longitudinal studies to assess the dynamicity of these beliefs, smoking behaviors, and successful quit attempts among those who have a willingness to cease smoking.

\section{CONCLUSION}

A significant proportion of participants have reported anti-smoking attitudes toward certain smoking methods while a minority did not have a conclusive attitude/belief. Besides, the pandemic crisis has impacted most current smokers' smoking habits and encouraged a noticeable proportion to consider smoking cessation. Considering the dynamicity of human health behaviors, a large-scale event like the current COVID-19 pandemic may exhibit significant effects that lead to adopting risky behaviors (e.g., initiation of smoking, increased smoking), or more healthy behaviors (e.g., quitting smoking, reducing consumption). Consequently, public health authorities and decision-makers should consider the double-edged effect of the COVID-19 pandemic on people's attitudes/beliefs toward smoking in order to maintain a more prominent anti-smoking attitude among people and to provide accessible quitting services on a wide range. Reliable antismoking actions and measures should be implemented and maintained especially in contexts where smoking prevalence is high and stressful events are prominent as these events may act as a spark for stress-induced smoking.

\section{LIST OF ABBREVIATIONS}

ACE2: Angiotensin-Converting Enzyme-2; CC: Combustible Cigarettes; COVID-19: Coronavirus Disease2019; EC: Electronic Cigarettes; EMR: Eastern Mediterranean Region; ICU: Intensive Care Unit; MPOWER: Monitor, Protect, Offer, Warn, Enforce, Raise; SARS-CoV-2: Severe Acute Respiratory SyndromeCoronavirus 2; WP: Waterpipe.

\section{DECLARATIONS}

Funding: This research project did not receive any specific grant from funding agencies in the public, commercial, or not-for-profit sectors

Conflict of interests: The authors have no conflicts of interest to declare that are relevant to the content of this article.

Ethical approval: The protocol of this study was reviewed and approved by the institutional review board at [anonymized for peer review] (Ref. Anonymized for peer review).

Consent to participate: All participants have provided an online written informed consent.

Consent for publication: Not Applicable

Availability of data: The dataset generated and analyzed in the current study is not publicly available due to compliance with institutional guidelines but it is available from the corresponding author on a reasonable request.

\section{REFERENCES}


[1] Al-Tammemi AB. The Battle Against COVID-19 in Jordan: An Early Overview of the Jordanian Experience. Front Public Heal 2020; 8: 188.

[2] Nicola M, Alsafi Z, Sohrabi C, et al. The socio-economic implications of the coronavirus pandemic (COVID-19): A review. Int J Surg 2020; 78: 185-193.

[3] Brooks SK, Webster RK, Smith LE, et al. Rapid Review The psychological impact of quarantine and how to reduce it: rapid review of the evidence. Lancet 2020; 395: 912-920.

[4] Al-Tammemi AB, Akour A, Alfalah L. Is It Just About Physical Health? An Online Cross-Sectional Study Exploring the Psychological Distress Among University Students in Jordan in the Midst of COVID-19 Pandemic. Front Psychol 2020; 11: 562213.

[5] Akour A, Al-Tammemi AB, Barakat M, et al. The Impact of the COVID-19 Pandemic and Emergency Distance Teaching on the Psychological Status of University Teachers: A Cross-Sectional Study in Jordan. Am J Trop Med Hyg 2020; 103: 2391-2399.

[6] Garboczy S, Szeman-Nagy A, Ahmad MS, et al. Health anxiety, perceived stress, and coping styles in the shadow of the COVID-19. BMC Psychol 2021; 9: 53.

[7] World Health Organization. Coronavirus (COVID-19) Dashboard. [Online/, https://covid19.who.int/ (2021, accessed 15 March 2021).

[8] Jordanian Ministry of Health. COVID-19 statistical report. [Online], https://corona.moh.gov.jo/en (2021, accessed 15 March 2021).

[9] Al-Tammemi AB, Tarhini Z, Akour A. A swaying between successive pandemic waves and pandemic fatigue: Where does Jordan stand? Ann Med Surg 2021; 65: 102298.

[10] Khatatbeh M. The Battle Against COVID-19 in Jordan: From Extreme Victory to Extreme Burden. Front Public Heal 2021; 8: 1038.

[11] Khatatbeh M. Efficacy of Nationwide Curfew to Encounter Spread of COVID-19: A Case From Jordan . Frontiers in Public Health 2020; 8: 394.

[12] Alrawashdeh HM, Al-Tammemi AB, Alzawahreh MK, et al. Occupational Burnout and Job Satisfaction Among Physicians in Times of COVID-19 Crisis: A Convergent Parallel Mixed-Method Study. BMC Public Health 2021; 21: 811.

[13] World Health Organization. Health Topics - Tobacco Facts. /Online/, https://www.who.int/healthtopics/tobacco\#tab=tab_1 (2021, accessed 2 February 2021).

[14] Strzelak A, Ratajczak A, Adamiec A, et al. Tobacco Smoke Induces and Alters Immune Responses in the Lung Triggering Inflammation, Allergy, Asthma and Other Lung Diseases: A Mechanistic Review. Int J Environ Res Public Health 2018; 15: 1033.

[15] World Health Organization. Tobacco Key Facts. [Online], https://www.who.int/news-room/factsheets/detail/tobacco (2020, accessed 15 March 2021).

[16] World Health Organization. Tobacco increases the poverty of individuals and families. /Online], https://www.who.int/tobacco/communications/events/wntd/2004/tobaccofacts_families/en/ (2004, accessed 15 March 2021).

[17] Shastri MD, Shukla SD, Chong WC, et al. Smoking and COVID-19: What we know so far. Respir Med 2021; 176: 106237.

[18] Vardavas CI, Nikitara K. COVID-19 and smoking: A systematic review of the evidence. Tob Induc Dis 2020; 18: 20. 
[19] Kalan ME, Ghobadi H, Taleb Z Ben, et al. COVID-19 and beliefs about tobacco use: an online cross-sectional study in Iran. Environ Sci Pollut Res. Epub ahead of print 2020. DOI: 10.1007/s11356-02011038-x.

[20] Patanavanich R, Glantz SA. Smoking Is Associated With COVID-19 Progression: A Meta-analysis. Nicotine Tob Res 2020; 22: 1653-1656.

[21] Farsalinos K, Barbouni A, Niaura R. Systematic review of the prevalence of current smoking among hospitalized COVID-19 patients in China: could nicotine be a therapeutic option? Intern Emerg Med 2020; 15: 845-852.

[22] Rentsch CT, Kidwai-Khan F, Tate JP, et al. Covid-19 Testing, Hospital Admission, and Intensive Care Among 2,026,227 United States Veterans Aged 54-75 Years. MedRxiv Prepr. Epub ahead of print 14 April 2020. DOI: 10.1101/2020.04.09.20059964.

[23] de Lusignan S, Dorward J, Correa A, et al. Risk factors for SARS-CoV-2 among patients in the Oxford Royal College of General Practitioners Research and Surveillance Centre primary care network: a cross-sectional study. Lancet Infect Dis 2020; 20: 1034-1042.

[24] Simons D, Shahab L, Brown J, et al. The association of smoking status with SARS-CoV-2 infection, hospitalization and mortality from COVID-19: a living rapid evidence review with Bayesian meta-analyses (version 7). Addiction. Epub ahead of print October 2020. DOI: 10.1111/add.15276.

[25] Alqahtani JS, Oyelade T, Aldhahir AM, et al. Prevalence, Severity and Mortality associated with COPD and Smoking in patients with COVID-19: A Rapid Systematic Review and Meta-Analysis. PLoS One 2020; 15: e0233147.

[26] Kayhan Tetik B, Gedik Tekinemre I, Taş S. The Effect of the COVID-19 Pandemic on Smoking Cessation Success. J Community Health 2020; 1-5.

[27] Safi M, Al-tahat J. Jordan smoking rates highest in world amid claims of big tobacco interference. The Guardian, https://www.theguardian.com/world/2020/jun/23/jordan-smoking-rates-highest-inworld-amid-claims-of-big-tobacco-interference (2020, accessed 2 February 2021).

[28] Burki TK. Tobacco control in Jordan. Lancet Respir 2019; 7: P386.

[29] World Population Review. Global Country Ranking by Smoking Rates. [Online], https://worldpopulationreview.com/country-rankings/smoking-rates-by-country (2021, accessed 2 February 2021).

[30] Campaign for Tobacco-Free Kids. Tobacco Control Laws - Jordan. [Online], https://www.tobaccocontrollaws.org/legislation/country/jordan/laws (2021, accessed 2 April 2021).

[31] World Bank Group. Overview of Tobacco Use, Tobacco Control Legislation, and Taxation in Jordan. [Online], https://elibrary.worldbank.org/doi/abs/10.1596/31954 (2019, accessed 16 April 2021).

[32] Bommelé J, Hopman P, Walters BH, et al. The double-edged relationship between COVID-19 stress and smoking: Implications for smoking cessation. Tob Induc Dis 2020; 18: :63.

[33] Hefler M, Gartner CE. The tobacco industry in the time of COVID-19: Time to shut it down? Tob Control 2020; 29: 245-246.

[34] Eisenberg SL, Eisenberg MJ. Smoking Cessation During the COVID-19 Epidemic. Nicotine Tob Res 2020; 22: 1664-1665.

[35] Kayhan Tetik B, Gedik Tekinemre I, Taş S. The Effect of the COVID-19 Pandemic on Smoking Cessation Success. J Community Health. Epub ahead of print 2020. DOI: 10.1007/s10900-020-00880-2.

[36] Vardavas CI, Nikitara K. COVID-19 and smoking: A systematic review of the evidence. Tob Induc Dis 2020; 18: :20. 
[37] Ho LLK, Li WHC, Cheung AT, et al. Impact of COVID-19 on the hong kong youth quitline service and quitting behaviors of its users. Int J Environ Res Public Health 2020; 17: 8397.

[38] Department of Statistics Jordan. Population Count. [Online], http://dosweb.dos.gov.jo/ (2020, accessed 16 April 2020).

[39] Yingst JM, Krebs NM, Bordner CR, et al. Tobacco Use Changes and Perceived Health Risks among Current Tobacco Users during the COVID-19 Pandemic. Int J Environ Res Public Health 2021; 18: 1795.

[40] Tsigaris P, Teixeira Da Silva JA. Smoking Prevalence and COVID-19 in Europe. Nicotine Tob Res 2020; 22: 1646-1649.

[41] Bar-Zeev Y, Shauly-Aharonov M, Lee H, et al. Changes in smoking behaviour and home-smoking rules during the initial COVID-19 lockdown period in Israel. Int J Environ Res Public Health 2021; 18: 1931.

[42] Soule EK, Mayne S, Snipes W, et al. Impacts of COVID-19 on electronic cigarette purchasing, use and related behaviors. Int J Environ Res Public Health 2020; 17: 6762.

[43] Jha P, Peto R. Global effects of smoking, of quitting, and of taxing tobacco. N Engl J Med 2014; 370: $60-68$.

[44] Yach D. Tobacco Use Patterns in Five Countries During the COVID-19 Lockdown. Nicotine Tob Res 2020; 22: 1671-1672.

[45] Ioannidis JPA, Jha P. Does the COVID-19 pandemic provide an opportunity to eliminate the tobacco industry? Lancet Glob Heal 2021; 9: e12-e13.

[46] Mistry SK, Ali AM, Rahman MA, et al. Changes in Tobacco Use Patterns During COVID-19 and Their Correlates among Older Adults in Bangladesh. Int $J$ Environ Res Public Health 2021; 18: 1779.

[47] Joyce AA, Styklunas GM, Rigotti NA, et al. Quit Experiences among Primary Care Patients Enrolled in a Smoking Cessation Pilot RCT Early in the COVID-19 Pandemic. Int J Environ Res Public Health 2021; 18: 1011.

[48] Kelly BC, Pawson M, Vuolo M. Beliefs on COVID-19 Among Electronic Cigarette Users: Behavioral Responses and Implications for COVID Prevention and E-Cigarette Interventions. J Drug Issues 2020; 51: $284-300$.

[49] Oakes JM, Fuchs RM, Gardner JD, et al. Nicotine and the renin-angiotensin system. Am J Physiol Regul Integr Comp Physiol 2018; 315: R895-R906.

[50] Yue X, Basting TM, Flanagan TW, et al. Nicotine Downregulates the Compensatory AngiotensinConverting Enzyme 2/Angiotensin Type 2 Receptor of the Renin-Angiotensin System. Ann Am Thorac Soc 2018; 15: S126-S127.

[51] Brake SJ, Barnsley K, Lu W, et al. Smoking Upregulates Angiotensin-Converting Enzyme-2 Receptor: A Potential Adhesion Site for Novel Coronavirus SARS-CoV-2 (Covid-19). Journal of clinical medicine; 9. Epub ahead of print March 2020. DOI: 10.3390/jcm9030841.

[52] Leung JM, Yang CX, Tam A, et al. ACE-2 expression in the small airway epithelia of smokers and COPD patients: implications for COVID-19. The European respiratory journal; 55. Epub ahead of print May 2020. DOI: 10.1183/13993003.00688-2020.

[53] Cai G. Bulk and single-cell transcriptomics identify tobacco-use disparity in lung gene expression of ACE2, the receptor of 2019-nCov. MedRxiv. Epub ahead of print 1 January 2020. DOI: 10.1101/2020.02.05.20020107.

[54] van Zyl-Smit RN, Richards G, Leone FT. Tobacco smoking and COVID-19 infection: Spotlight. Lancet Respir Med 2020; 8: 664-665. 
[55] Alla F, Berlin I, Nguyen-Thanh V, et al. Tobacco and COVID-19: a crisis within a crisis? Can J Public Heal 2020; 111: 995-999.

[56] Alagaili AN, Briese T, Amor NMS, et al. Waterpipe smoking as a public health risk: Potential risk for transmission of MERS-CoV. Saudi J Biol Sci 2019; 26: 938-941.

[57] Urkin J, Ochaion R, Peleg A. Hubble Bubble Equals Trouble: The Hazards of Water Pipe Smoking. Sci World J 2006; 6: 817863.

[58] Munckhof WJ, Konstantinos A, Wamsley M, et al. A cluster of tuberculosis associated with use of a marijuana water pipe. Int J Tuberc Lung Dis 2003; 7: 860-865.

[59] El Barrawy MA, Morad MI, Gaber M. Role of Helicobacter pylori in the genesis of gastric ulcerations among smokers and nonsmokers. East Mediterr Heal J 1997; 3: 316-321.

[60] Habib M, Mohamed MK, Abdel-Aziz F, et al. Hepatitis C virus infection in a community in the Nile Delta: Risk factors for seropositivity. Hepatology 2001; 33: 248-253.

[61] Knishkowy B, Amitai Y. Water-Pipe (Narghile) Smoking: An Emerging Health Risk Behavior. Pediatrics 2005; 116: e113 LP-e119.

[62] Szyper-Kravitz M, Lang R, Manor Y, et al. Early Invasive Pulmonary Aspergillosis in a Leukemia Patient Linked to Aspergillus Contaminated Marijuana Smoking. Leuk Lymphoma 2001; 42: 1433-1437.

[63] Shekhar S, Hannah-Shmouni F. Hookah smoking and COVID-19: call for action. Can Med Assoc J 2020; 192: E462.

[64] Reddy RK, Charles WN, Sklavounos A, et al. The effect of smoking on COVID-19 severity: A systematic review and meta-analysis. J Med Virol 2021; 93: 1045-1056.

[65] Barakat M, Assaf AM, Al-Qudah R, et al. Perception of adults toward electronic cigarettes: a cross-sectional study from Jordan. Prim Health Care Res Dev 2021; 22: 1-9.

[66] Barakat M, Jirjees F, Al-Tammemi AB, et al. The Era of E-Cigarettes: A Cross-Sectional Study of Vaping Preferences, Reasons for Use and Withdrawal Symptoms Among Current E-Cigarette Users in the United Arab Emirates. J Community Health. Epub ahead of print 2021. DOI: 10.1007/s10900-021-00967-4.

[67] Barakat MM, Al-Qudah RA, Alfayoumi I, et al. Electronic cigarettes' withdrawal severity symptoms among users during intermittent fasting: a cross-sectional study. Addict Sci Clin Pract 2021; 16: 10.

[68] Karasneh R, Al-Azzam S, Nusair M, et al. Perceptions, symptoms, and practices of electronic cigarette users: Descriptive analysis and validation of Arabic short form vaping consequences questionnaire. PLoS One 2021; 16: e0245443.

[69] Institute for Global Tobacco Control. E-cigarettes Regulations: A Policy Scan. [Online], https://www.globaltobaccocontrol.org/e-cigarette/jordan (2020, accessed 3 March 2020).

[70] Abdel-Qader DH, Al Meslamani AZ. Knowledge and Beliefs of Jordanian Community Toward Ecigarettes: A National Survey. J Community Health. Epub ahead of print 2020. DOI: 10.1007/s10900-02000896-8.

[71] Al-Balas HI, Al-Balas M, Al-Balas H, et al. Electronic Cigarettes Prevalence and Awareness Among Jordanian Individuals. J Community Health. Epub ahead of print 2020. DOI: 10.1007/s10900-020-00904-x. 